\title{
Discrete-Time Sliding Mode Control for Uncertain Networked System Subject to Time Delay
}

\author{
Saulo C. Garcia, Marcelo C. M. Teixeira, José Paulo F. Garcia, \\ Uiliam N. L. T. Alves, and Jean M. S. Ribeiro \\ Control Research Laboratory, Department of Electrical Engineering, São Paulo State University (UNESP), \\ 15385-000 Ilha Solteira, SP, Brazil \\ Correspondence should be addressed to José Paulo F. Garcia; jpaulo@dee.feis.unesp.br
}

Received 6 June 2014; Revised 4 August 2014; Accepted 6 August 2014

Academic Editor: Zhan Shu

Copyright (C) 2015 Saulo C. Garcia et al. This is an open access article distributed under the Creative Commons Attribution License, which permits unrestricted use, distribution, and reproduction in any medium, provided the original work is properly cited.

We deal with uncertain systems with networked sliding mode control, subject to time delay. To minimize the degenerative effects of the time delay, a simpler format of state predictor is proposed in the control law. Some ultimate bounded stability analyses and stabilization conditions are provided for the uncertain time delay system with proposed discrete-time sliding mode control strategy. A numerical example is presented to corroborate the analyses.

\section{Introduction}

Networked control system (NCS) is a very convenient strategy of control for industrial plants, where actuators and machines are located in rustic setting [1]. In those environments, a digital device, which computes the control signals, is susceptible to degeneration. Many other advantages, such as simple installation, great flexibility, and low cost, make the NCS a strategy widely used and researched [2]. However, despite these advantages, NCS implies the need of data acquisition, conversion of analog signal in digital form, and processing the data to generate the control signal. These steps may cause time delays of several sampling periods, which tend to deteriorate the system performance [3]. Many control strategies that are robust with respect to parametric variations and nonlinearities of the plant exhibit great sensitivity when delays are present, losing all their features of robustness. Due to this and other problems and high control utilization, NCS has significantly attracted research communities with important results for numerous control methods such as fuzzy control, neural control, adaptive control, sliding mode control, optimal control techniques, and many more techniques [4-11].

In particular, the strategy sliding mode control (SMC) $[12,13]$ is more sensitive to time delay. The main advantage of SMC is its robustness with respect to matched uncertainties
[14]. By using a high speed switching control law in order to take the states trajectory to a sliding surface, if the states used are delayed, the control law may not direct the states to this surface, which can also generate performance loss or even lead system to instability. The damage caused by delays to sliding mode control motivates several studies with important results, including SMC performed by NCS [15-18].

In this paper we deal with uncertain system with discretetime sliding mode control (DSMC) performed with NCS subject to time delay. To minimize the degenerative effects of delay, a discrete-time state predictor in simplified form used in conjunction with DSMC is proposed. Some ultimate bounded stability analyses and stabilization conditions are provided for the uncertain time delay system with proposed discrete-time sliding mode control strategy. A numerical example is presented to corroborate the results shown in the analyses.

\section{Systems with Time Delay due to NCS}

Consider the following uncertain discrete-time system:

$$
x_{k+1}=\Phi x_{k}+\Gamma v_{k}+f_{k},
$$

where $x_{k} \in \mathfrak{R}^{n}$ is the state vector, $v_{k} \in \mathfrak{R}^{m}$ is the control vector, and $f_{k} \in \mathfrak{R}^{n}$ is the bounded uncertainties vector. The 


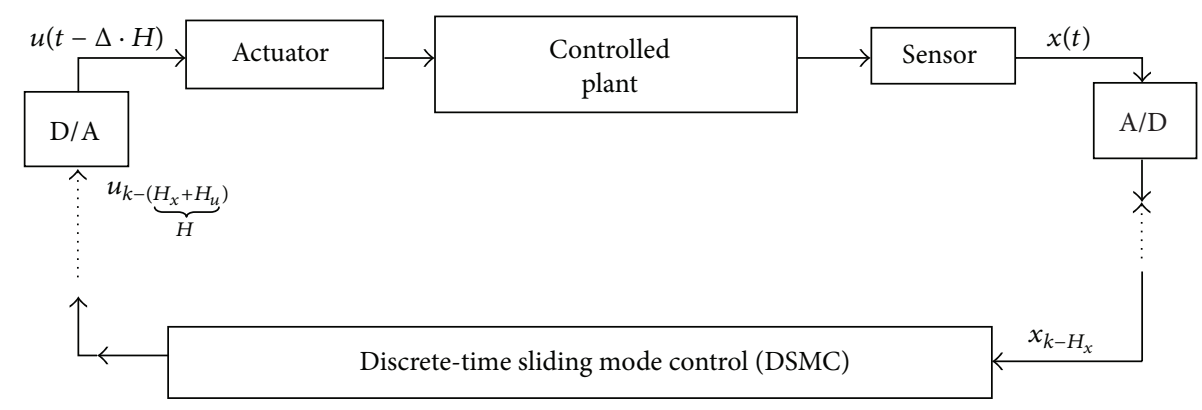

FIGURE 1: NCS representation in direct structure.

matrices $\Phi \in \mathfrak{R}^{n x n}$ and $\Gamma \in \mathfrak{R}^{n x m}$ are constants with nominal values of the plant, which is controllable.

The discrete-time law is given by

$$
v(t)=v_{k}=F x_{k} ; \quad k \Delta \leq t<(k+1) \Delta,
$$

where $\Delta$ is the sampling period and $F \in \mathfrak{R}^{m x n}$ is the state feedback gain matrix, whose design can be done through various methods. For the design of matrix $F$, in this paper, we analyze the stability robustness of the technique discretetime sliding mode control (DSMC) performed by NCS.

As represented in Figure 1, when the control of the system is performed by a network in direct structure, the presence of delay due to the time required for analog and digital conversion is very common for receiving, processing, and transmission of data.

When the delay is directly related to the time required for state vector signal transmission, we have

$$
v_{k}=F x_{k-H_{x}}
$$

where $H_{x}$ is the number of periods of delayed sampling state vector. Thus, we have the equivalence to a system with delayed control signal as

$$
x_{k+1}=\Phi x_{k}+\Gamma u_{k-H_{x}}+f_{k},
$$

where $u_{k-H_{x}}=F x_{k-H_{x}}$.

When the delay is due to the time required for control signal transmission we have

$$
x_{k+1}=\Phi x_{k}+\Gamma u_{k-H_{u}}+f_{k},
$$

where $H_{u}$ is the number of sampling periods of the delayed control signal.

Usually these time delays occur simultaneously in the NCS so that

$$
u_{k-H}=F x_{k-H},
$$

where $H=H_{x}+H_{u}$. So, we have the following system:

$$
x_{k+1}=\Phi x_{k}+\Gamma u_{k-H}+f_{k} \text {. }
$$

If the sampling time is $\Delta$, the control time delay in the continuous time system is $\Delta \cdot H$. It is known that time delays in control signals degrade system performance [6-9]. Specifically, sliding mode control is more sensitive to these types of failures. In the next section, the use of a simpler format of state predictor to minimize the damaging effects of the time delay is proposed.

\section{State Predictor}

For the system (7) the following control law is proposed:

$$
u_{k}=F \widehat{x}_{k}
$$

where the predictive state vector $\hat{x}_{k}$ is an estimate of actual state vector at sample time $k+H$, that is, $x_{k+H}$.

In Xia et al. [19], the authors present a predictor of states for discrete-time systems with control time delay given by

$$
\widehat{x}_{k}=\Phi^{H} x_{k}+\sum_{i=-H+1}^{0} \Phi^{-i} \Gamma u(k-1+i) .
$$

By (9), we observe that to obtain the predicted states, several past samples of the control signal are needed and, for each sampling period, many calculations are required. We therefore propose predictors of states that require neither sampling control signal nor calculations that cause large processing time.

Consider the system (7) with the control law (8) and assume uncertainties are zero; that is, $f_{k}=0$. So, $\widehat{x}_{k}=x_{k+H}$ and $u_{k-H}=F x_{k}$. Then,

$$
\begin{aligned}
x_{k+1} & =\Phi x_{k}+\Gamma \underbrace{F x_{(k+H)-H}}_{u_{k-H}}=(\Phi+\Gamma F) x_{k} ; x_{k+2} \\
& =(\Phi+\Gamma F) x_{k+1}=(\Phi+\Gamma F)(\Phi+\Gamma F) x_{k} \\
& =(\Phi+\Gamma F)^{2} x_{k},
\end{aligned}
$$

and for the sample time $k+H$ we have

$$
x_{k+H}=\underbrace{(\Phi+\Gamma F)^{H}}_{\Psi} x_{k} .
$$

By (11), we define the predicted state vector as

$$
\widehat{x}_{k}=\Psi^{(\widehat{H})} x_{k},
$$

where $\Psi=(\Phi+\Gamma F)$ is a stable matrix and $\widehat{H}$ is an estimate value of the actual delay $H$. Note that

$$
x_{k}=\Psi^{(-\widehat{H})} \widehat{x}_{k} .
$$

It should be noted that, in the presence of uncertainties, that is, $f_{k} \neq 0$ and $H \neq \widehat{H}$, the predictor (12) is not accurate.

The following proposition deals with the dynamics of the proposed predictor (12). 
Proposition 1. Consider the uncertain time delay system (7) with the control law (8) and the state predictor (12). Then the dynamics of predictive states are given by

$$
\widehat{x}_{k+1}=\bar{\Phi} \widehat{x}_{k}+\bar{\Gamma} \bar{u}_{k}+\Psi^{(\widehat{H})} \widetilde{e}_{k},
$$

where $\bar{\Phi}=\Psi^{(\widehat{H})} \Phi \Psi^{(-\widehat{H})}, \bar{\Gamma}=\Psi^{(\widehat{H})} \Gamma, \bar{u}_{k}=F \Psi^{(-\widehat{H})} \widehat{x}_{k}=\bar{F} \widehat{x}_{k}$, $\widetilde{e}_{k}=f_{k}-\Gamma F e_{k}$, and $e_{k}=x_{k}-\widehat{x}_{k-\widetilde{H}}$.

Proof. Due to uncertainties, we have $\widehat{x}_{k-H} \neq x_{k}$. We define the error vector as

$$
\begin{aligned}
& e_{k}=x_{k}-\widehat{x}_{k-H}, \\
& \widehat{x}_{k-H}=x_{k}-e_{k} .
\end{aligned}
$$

In (12), at sampling period $k+1$, it follows that

$$
\widehat{x}_{k+1}=\Psi^{(\widehat{H})} x_{k+1} .
$$

Substituting (7) into (16), we have

$$
\widehat{x}_{k+1}=\Psi^{(\widehat{H})}\left(\Phi x_{k}+\Gamma u_{k-H}+f_{k}\right) .
$$

Using (8) and (13) into (17) we obtain

$$
\widehat{x}_{k+1}=\Psi^{(\widehat{H})}(\Phi \underbrace{\Psi^{(-\widehat{H})} \widehat{x}_{k}}_{x_{k}}+\Gamma \underbrace{F \widehat{x}_{k-H}}_{u_{k-H}}+f_{k}) .
$$

Substituting (15) into (18) and rearranging, we get

$$
\widehat{x}_{k+1}=\bar{\Phi} \widehat{x}_{k}+\bar{\Gamma} \bar{u}_{k}+\Psi^{(\widehat{H})} \widetilde{e}_{k},
$$

where $\bar{\Phi}=\Psi^{(\widehat{H})} \Phi \Psi^{(-\widehat{H})}, \bar{\Gamma}=\Psi^{(\widehat{H})} \Gamma, \bar{u}_{k}=F \Psi^{(-\widehat{H})} \widehat{x}_{k}=\bar{F} \widehat{x}_{k}$, and $\widetilde{e}_{k}=f_{k}-\Gamma F e_{k}$.

Note 1. The predictive state vector $\widehat{x}_{k}$ of (19) has the same closed-loop dynamics of the actual state vector $x_{k}$. It can be seen by substituting (8) and (12) into the system (7):

$$
x_{k+1}=\Phi x_{k}+\Gamma F \widehat{x}_{k-H}+f_{k} .
$$

Considering (15) and the matrix $\Psi=(\Phi+\Gamma F)$, we obtain the closed-loop dynamics of the actual state vector

$$
x_{k+1}=\underbrace{(\Phi+\Gamma F)}_{\Omega_{M F}=\Psi} x_{k}+\widetilde{e}_{k} .
$$

Also, by (19), the closed-loop dynamics of the predictive state vector $\widehat{x}_{k}$ are

$$
\widehat{x}_{k+1}=\underbrace{[\Psi^{(\widehat{H})} \underbrace{(\Phi+\Gamma F)}_{\Psi} \Psi^{(-\widehat{H})}]}_{\bar{\Omega}_{M F}=\Psi} \widehat{x}_{k}+\Psi^{(\widehat{H})} \widetilde{e}_{k} .
$$

Comparing (21) with (22) it can be noted that the matrices $\bar{\Omega}_{M F}$ and $\Omega_{M F}$ are both equal to $\Psi$. Thus, we conclude that the dynamics of the predictive state vector $\widehat{x}_{k}$ are the same as actual state vector $x_{k}$.
Note 2. In (21) and (22) it can be seen that, with the control (8) and state predictor (12), the influence of uncertainties in the dynamics of the system increases with the presence of the time delay due to the prediction error $\widetilde{e}_{k}=f_{k}-\Gamma F e_{k}$. According to control strategy adopted for the calculation of gain matrix $F$, there will be greater or lesser influence on system performance.

In the next section, the stability robustness will be analyzed specifically for discrete-time sliding mode control (DSMC).

\section{Discrete-Time Sliding Mode Control (DSMC) via NCS with Delay}

In this work, the proposed DSMC law can be of the form

$$
u(t)=u_{k}=u_{k}^{\mathrm{eq}}+u_{k}^{N}, \quad k \Delta \leq t<(k+1) \Delta,
$$

where $u_{k}^{\text {eq }} \in \Re^{m}$ is the equivalent control vector, which establishes the system dynamics in sliding mode, and $u_{k}^{N} \epsilon$ $\mathfrak{R}^{m}$ is the control signal vector that takes the trajectory of states to the sliding surface. In sliding mode, the system becomes less sensitive to certain classes of disturbances and uncertainty [14].

4.1. Design of DSMC with Predictive States. The equivalent control $u_{k}^{\text {eq }}$ is primarily designed. Later, the design of $u_{k}^{N}$ is performed.

A linear discrete-time sliding surface is defined as

$$
S_{k}=G x_{k}
$$

where the gain matrix $G \in \mathfrak{R}^{m x n}$ is designed such that the sliding trajectory has desired dynamics. The equivalent control is obtained with the following sliding condition:

$$
S_{k+1}=S_{k}
$$

Disregarding the uncertainties and substituting (7) and (24) into (25) we have

$$
\begin{gathered}
G \Phi x_{k}+G \Gamma u_{k-H}^{\mathrm{eq}}=G x_{k}, \\
u_{k-H}^{\mathrm{eq}}=-(G \Gamma)^{-1} G(\Phi-I) x_{k} .
\end{gathered}
$$

Thus, for the sample period $k$ we have

$$
u_{k}^{\mathrm{eq}}=F_{e} x_{k+H} .
$$

Using the predictor $x_{k+H}=\widehat{x}_{k}$ defined in (12), we obtain

$$
u_{k}^{\mathrm{eq}}=F_{e} \widehat{x}_{k} \text {, }
$$

where $F_{e}=-(G \Gamma)^{-1} G(\Phi-I) e G \Gamma$ is a nonsingular matrix.

Now, the control law $u_{k}^{N}$ is designed. Consider the following Lyapunov function:

$$
V_{k}=S_{k}^{T} S_{k} .
$$


The control law $u_{k}^{N}$ should provide to the system the condition of attractiveness to the sliding mode. For this to occur, the following condition of attractiveness must be satisfied:

$$
V_{k+1}<V_{k}
$$

By (29), it follows that (30) becomes

$$
S_{k+1}^{T} S_{k+1}<S_{k}^{T} S_{k}
$$

Defining [20],

$$
\Delta S_{k+1}=S_{k+1}-S_{k}
$$

Disregarding the uncertainties and substituting (7) and (24) into (32), we have

$$
\Delta S_{k+1}=G \Phi x_{k}+G \Gamma u_{k-H}^{\mathrm{eq}}+G \Gamma u_{k-H}^{N}-G x_{k} .
$$

By replacing $u_{k-H}^{\mathrm{eq}},(26)$, into (33) comes

$$
\Delta S_{k+1}=G \Gamma u_{k-H}^{N} .
$$

Replacing $S_{k+1}=S_{k}+\Delta S_{k+1}$ in condition (31) we find

$$
\left\|S_{k}+G \Gamma u_{k-H}^{N}\right\|<\left\|S_{k}\right\|,
$$

where $\left\|S_{k}\right\|$ is the Euclidian norm of vector $S_{k} \in \Re^{m}$.

A control law that satisfies the attractiveness condition (35) is defined as

$$
u_{k}^{N}=F_{n} \widehat{x}_{k}
$$

where $\widehat{x}_{k}=x_{k+H}, F_{n}=-(G \Gamma)^{-1} \beta G$, and $\beta \in \mathfrak{R}^{m x m}$ is a diagonal matrix with $0<\beta_{i i}<1, i=1, \ldots, m$. Thus, the control law $u_{k}=u_{k}^{\text {eq }}+u_{k}^{N}$ becomes

$$
u_{k}=F \widehat{x}_{k}
$$

with $F=F_{e}+F_{n}$.

4.2. Robustness Analysis. This section analyzes the stability robustness of the system (7) in the following proposition.

Proposition 2. Consider the uncertain time delay system (7), with control law (37) and (12). The attractiveness condition (30) is satisfied if $\left\|G x_{k}\right\|>\left\|G \widetilde{e}_{k}\right\|$, with $\widetilde{e}_{k}=f_{k}-\Gamma F\left(x_{k}-\widehat{x}_{k-H}\right)$.

Proof. Taking into account the uncertainties, that is, $f_{k} \neq 0$ and $H \neq \widehat{H}$, (32) becomes

$$
\Delta S_{k+1}=G\left(\Phi x_{k}+\Gamma u_{k-H}+f_{k}\right)-G x_{k}
$$

Substituting (37), (28), and (36) into (38), one comes to

$$
\Delta S_{k+1}=-\beta G x_{k}+G \widetilde{e}_{k}
$$

where $\tilde{e}_{k}=f_{k}-\Gamma F e_{k}$. Substituting (39) into (30), using (32) and considering $\beta_{i i}=\bar{\beta} \in \mathfrak{R}^{+}, i=1, \ldots, m$, we have

$$
2(1-\bar{\beta}) S_{k}{ }^{T} G \widetilde{e}_{k}<\left[1-(1-\bar{\beta})^{2}\right]\left\|S_{k}\right\|^{2}-\left\|G \widetilde{e}_{k}\right\|^{2} \text {. }
$$

If $0<\bar{\beta}<1.0$, so $2>2(1-\bar{\beta})>0$. Thus, it is possible to rearrange condition (40) as follows:

$$
S_{k}^{T} G \widetilde{e}_{k}<\varsigma\left\|S_{k}\right\|^{2}-\bar{\varsigma}\left\|G \widetilde{e}_{k}\right\|^{2}
$$

with $\varsigma=\left(1-(1-\bar{\beta})^{2}\right) / 2(1-\bar{\beta})$ and $\bar{\varsigma}=1 / 2(1-\bar{\beta})$. Considering that $0<\bar{\beta}<1,0$, so $(\bar{\varsigma} / \varsigma)>1,0$. With this, it can be seen, by (41), that the condition that satisfies the cases where $S_{k}{ }^{T} G \widetilde{e}_{k}>$ 0 and $S_{k}^{T} G \widetilde{e}_{k}<0$ is the following:

$$
\frac{\left\|S_{k}\right\|}{\left\|G \widetilde{e}_{k}\right\|}>\sqrt{\frac{\bar{\varsigma}}{\varsigma}}>1,0 .
$$

Therefore, to satisfy (41) the norm $\left\|S_{k}\right\|=\left\|G x_{k}\right\|$ must be greater than $\left\|G \widetilde{e}_{k}\right\|$.

Note 3. From Proposition 2 it follows that, when the uncertain time delay system (7) is in steady state, its states remain in the vicinity of the ideal equilibrium point (origin). This fact implies the concept "ultimate bounded stability" [21].

Proposition 3. Consider the uncertain time delay system (7) with the control law (37) and that attractiveness condition (30) is satisfied. Also, the actual number of delayed samples $H$ is unknown and the uncertainties are bounded; that is, $\left\|f_{k}\right\| \leq$ $d_{\max }<\infty$ for all values of $k$. So, in steady state the norm $\left\|\widetilde{e}_{k}\right\|$ will be also bounded; that is, $\lim _{k \rightarrow \infty}\left\|\widetilde{e}_{k}\right\|=\bar{b}_{\max }<\infty$, with $\tilde{e}_{k}=f_{k}-\Gamma F\left(x_{k}-\widehat{x}_{k-H}\right)$.

Proof. Consider the system (7) with $f_{k} \neq 0$ and the control law (37) with predictive state vector (12). So

$$
x_{k+1}=\Phi x_{k}+\Gamma F \widehat{x}_{k-H}+f_{k} .
$$

With $\widehat{x}_{k-H}=x_{k}-e_{k}$ we have

$$
\begin{aligned}
x_{k+1} & =\Phi x_{k}+\Gamma F\left(x_{k}-e_{k}\right)+f_{k} \\
& =(\Phi+\Gamma F) x_{k}+\left(f_{k}-\Gamma F e_{k}\right) .
\end{aligned}
$$

For $\Psi=\Phi+\Gamma F$ and $\widetilde{e}_{k}=f_{k}-\Gamma F e_{k}$ we have

$$
\begin{aligned}
x_{k+1} & =\Psi x_{k}+\widetilde{e}_{k} ; \\
x_{k+2} & =\Psi x_{k+1}+\widetilde{e}_{k+1}=\Psi \underbrace{\left(\Psi x_{k}+\widetilde{e}_{k}\right)}_{x_{k+1}}+\widetilde{e}_{k+1} \\
& =\Psi^{2} x_{k}+\Psi \widetilde{e}_{k}+\widetilde{e}_{k+1} ; \\
x_{k+3} & =\Psi^{2} x_{k+1}+\Psi \widetilde{e}_{k+1}+\widetilde{e}_{k+2} \\
& =\Psi^{2} \underbrace{\left(\Psi x_{k}+\widetilde{e}_{k}\right)}_{x_{k+1}}+\Psi \widetilde{e}_{k+1}+\widetilde{e}_{k+2} \\
& =\Psi^{3} x_{k}+\Psi^{2} \widetilde{e}_{k}+\Psi \widetilde{e}_{k+1}+\widetilde{e}_{k+2} ;
\end{aligned}
$$

and for the sample time $k+H$ we have

$$
\begin{aligned}
x_{k+H}= & \Psi^{H} x_{k}+\Psi^{(H-1)} \widetilde{e}_{k}+\Psi^{(H-2)} \widetilde{e}_{k+1} \\
& +\Psi^{(H-3)} \widetilde{e}_{k+2}+\cdots+\widetilde{e}_{k+(H-1)} .
\end{aligned}
$$


Thus, by (46), the predictive state vector $\bar{x}_{k}=x_{k+H}$ is accurately obtained by the equation

$$
\bar{x}_{k}=\Psi^{(H)} x_{k}+\sum_{i=0}^{H-1} \Psi^{-[i-(H-1)]}\left(\widetilde{e}_{k+i}\right) .
$$

The actual state vector is obtained as $x_{k}=\bar{x}_{k-H}$. So by (47) we have

$$
\bar{x}_{k-H}=\Psi^{(H)} x_{k-H}+\sum_{i=0}^{H-1} \Psi^{-[i-(H-1)]}\left(\widetilde{e}_{k-H+i}\right) .
$$

We defined the predictor (not exact) as $\widehat{x}_{k}=\Psi^{\widehat{H}} x_{k}$ and the error is given by $e_{k}=x_{k}-\widehat{x}_{k-H}$. Then, with (48) and considering that $\tilde{e}_{k}=f_{k}-\Gamma F e_{k}$, we have

$$
\begin{aligned}
e_{k}= & \sum_{i=0}^{H-1} \Psi^{-[i-(H-1)]}\left[f_{k-H+i}-(\Gamma F)\left(e_{k-H+i}\right)\right] \\
& +\Psi_{f} x_{k-H},
\end{aligned}
$$

where $\Psi_{f}=\Psi^{(H)}-\Psi^{(\widehat{H})}$. For the sampling period $k+N$ becomes

$$
\begin{aligned}
e_{k+N}+\left[\sum_{i=0}^{H-1} \Psi^{-[i-(H-1)]}(\Gamma F)\left(e_{k-H+i+N}\right)\right] \\
=\left[\sum_{i=0}^{H-1} \Psi^{-[i-(H-1)]}\left(f_{k-H+i+N}\right)\right]+\Psi_{f}\left(x_{k-H+N}\right) .
\end{aligned}
$$

It is easy to deduce that $x_{k-H+1}=\Psi x_{k-H}+\widetilde{e}_{k-H}$. So for sampling period $k-H+N$ we have

$$
\begin{aligned}
x_{k-H+1} & =\Psi x_{k-H}+\widetilde{e}_{k-H} ; \\
x_{k-H+2} & =\Psi \underbrace{x_{k-H+1}}_{\Psi x_{k-H}+\widetilde{e}_{k-H}}+\widetilde{e}_{k-H+1} \\
& =\Psi^{2} x_{k-H}+\Psi \widetilde{e}_{k-H}+\widetilde{e}_{k-H+1} ; \\
& \vdots \\
x_{k-H+N} & =\Psi^{(N)} x_{k-H}+\sum_{i=0}^{N-1} \Psi^{-[i-(N-1)]}\left(\widetilde{e}_{k-H+i}\right) .
\end{aligned}
$$

Thus, by (51), (50) can be rewritten as

$$
\begin{aligned}
& e_{k+N}+\sum_{i=0}^{H-1} \Psi^{-[i-(H-1)]}(\Gamma F)\left(e_{k+N-H+i}\right) \\
& =\sum_{i=0}^{H-1} \Psi^{-[i-(H-1)]}\left(f_{k+N-H+i}\right)+\left(\Psi_{f} \Psi^{(N)}\right)\left(x_{k-H}\right) \\
& +\Psi_{f}[\sum_{i=0}^{N-1} \Psi^{-[i-(N-1)]} \underbrace{\left(\widetilde{e}_{k-H+i}\right)}_{\left[\left(f_{k-H+i}\right)-(\Gamma F)\left(e_{k-H+i}\right)\right]}] e_{k+N} \\
& +\sum_{i=0}^{H-1} \Psi^{-[i-(H-1)]}(\Gamma F)\left(e_{k+N-H+i}\right) \\
& =\sum_{i=0}^{H-1} \Psi^{-[i-(H-1)]}\left(f_{k+N-H+i}\right) \\
& +\left(\Psi_{f} \Psi^{(N)}\right)\left(x_{k-H}\right)+\Psi_{f} \sum_{i=0}^{N-1} \Psi^{-[i-(N-1)]}\left(f_{k-H+i}\right) \\
& -\Psi_{f} \sum_{i=0}^{N-1} \Psi^{-[i-(N-1)]}\left[(\Gamma F)\left(e_{k-H+i}\right)\right] e_{k+N} \\
& +\sum_{i=0}^{H-1} \Psi^{-[i-(H-1)]}(\Gamma F)\left(e_{k+N-H+i}\right) \\
& +\Psi_{f} \sum_{i=0}^{N-1} \Psi^{-[i-(N-1)]}\left[(\Gamma F)\left(e_{k-H+i}\right)\right] \\
& =\left(\Psi_{f} \Psi^{(N)}\right)\left(x_{k-H}\right)+\sum_{i=0}^{H-1} \Psi^{-[i-(H-1)]}\left(f_{k+N-H+i}\right) \\
& +\Psi_{f} \sum_{i=0}^{N-1} \Psi^{-[i-(N-1)]}\left(f_{k-H+i}\right) \text {. }
\end{aligned}
$$

The norm of $f_{k}$ is bounded. Suppose for simplicity, without loss of generality, that this vector is constant, $f_{k}=f$, such that $\|f\|=d_{\max }$. So, (52) becomes

$$
\begin{aligned}
e_{k+N} & +\sum_{i=0}^{H-1} \Psi^{-[i-(H-1)]}(\Gamma F)\left(e_{k-H+i+N}\right) \\
& +\Psi_{f} \sum_{j=0}^{N-1} \Psi^{-[j-(N-1)]}(\Gamma F)\left(e_{k-H+j}\right) \\
= & \left(\Psi_{f} \Psi^{(N)}\right)\left(x_{k-H}\right) \\
& +\left(\Psi_{f} \sum_{j=0}^{N-1} \Psi^{-[j-(N-1)]}+\sum_{i=0}^{H-1} \Psi^{-[i-(H-1)]}\right)(f) .
\end{aligned}
$$


The closed-loop matrix $\Psi$ is designed to be stable, that is, all its eigenvalues are inside in the unit circle. Then in the second member of (53) we have

$$
\begin{gathered}
\lim _{k, N \rightarrow \infty}\left\{\left(\Psi_{f} \sum_{j=0}^{N-1} \Psi^{-[i-(N-1)]}+\sum_{i=0}^{H-1} \Psi^{-[i-(H-1)]}\right)\right\}=\Upsilon, \\
\|\Upsilon\|<\infty \\
\lim _{k, N \rightarrow \infty}\left\{\left(\Psi_{f} \Psi^{(N)}\right)\right\}=0 .
\end{gathered}
$$

So for (53) to be satisfied, in the first member of this equation, $\lim _{k \rightarrow \infty} e_{k}$ must be also a constant. Thus, it can be concluded that

$$
\begin{aligned}
& \lim _{k, N \rightarrow \infty}\left\|e_{k}\right\|=b_{\max }<\infty, \\
& \lim _{k, N \rightarrow \infty}\left\|\widetilde{e}_{k}\right\|=\bar{b}_{\max }<\infty .
\end{aligned}
$$

Note 4. With $f_{k}=f$, by Proposition 3, we have that $\lim _{k \rightarrow \infty} e_{k}=e$ and $\|e\|=b_{\max }<\infty$. For stability, the attractiveness condition $\left\|x_{k}\right\|>\left\|\widetilde{e}_{k}\right\|=\left\|\left(f_{k}-\Gamma F e_{k}\right)\right\|$ is maintained until the system enters in the steady state, that is, until the state trajectory enters the neighborhood of the origin. The value of norm $\|e\|=b_{\max }$ is established, as can be seen in (53) and (54), by

$$
\left(\Psi_{f} \sum_{j=0}^{N-1} \Psi^{-[j-(N-1)]}+\sum_{i=0}^{H-1} \Psi^{-[i-(H-1)]}\right)(f) .
$$

Then, the higher the value of $H$, the higher the value of $\left\|\left(f_{k}-\Gamma F e_{k}\right)\right\|$. This imposes a limit on the maximum number of delayed samples $H_{\text {max }}$, in which it is possible to satisfy the attractiveness condition of sliding mode control.

Proposition 4. Consider the uncertainties with bounded norm; that is, $\left\|f_{k}\right\| \leq d_{\max }<\infty$, for all $k$ and $\lim _{k \rightarrow \infty}\left\|\widetilde{e}_{k}\right\|=$ $\bar{b}_{\max }<\infty$, where $\widetilde{e}_{k}=f_{k}-\Gamma F\left(x_{k}-\widehat{x}_{k-H}\right)$. If the attractiveness condition (30) is satisfied, the system (7) will be uniformly ultimately bounded with control law (37), (8), and (12).

Proof. This proof is a version of result and terminology presented in [19]. Consider a linear transformation for the uncertain time delay system (7) of the form

$$
\begin{aligned}
& T x_{k+1} \\
& \quad=\left[\begin{array}{l}
z_{1_{k+1}} \\
z_{2_{k+1}}
\end{array}\right]=\left[\begin{array}{ll}
\bar{\Phi}_{11} & \bar{\Phi}_{12} \\
\Phi_{21} & \bar{\Phi}_{22}
\end{array}\right]\left[\begin{array}{l}
z_{1_{k}} \\
z_{2_{k}}
\end{array}\right]+\left[\begin{array}{c}
\overline{0} \\
\bar{\Gamma}_{2}
\end{array}\right] u_{k-H}+\left[\begin{array}{l}
\bar{f}_{1_{k}} \\
\bar{f}_{2_{k}}
\end{array}\right],
\end{aligned}
$$

where $z_{1_{k}} \in \Re^{n-m}, z_{2_{k}} \in \Re^{m}, \bar{f}_{1_{k}} \in \Re^{n-m}$ is the unmatched uncertainty vector; $\bar{f}_{2_{k}} \in \Re^{m}$ is the matched uncertainty vector; $\bar{\Phi}_{11}, \bar{\Phi}_{12}, \bar{\Phi}_{21}, \bar{\Phi}_{22}$, and $\bar{\Gamma}_{2}$ are constant matrices with appropriate dimensions; and $\overline{0} \in \mathfrak{R}^{(n-m) x(m)}$ is the zero matrix. Note that, because the uncertainties $f_{k}$ has bounded norm, so $\left\|\bar{f}_{1_{k}}\right\| \leq f_{1}<\infty$ and $\left\|\bar{f}_{2_{k}}\right\| \leq f_{2}<\infty$ for all $k$.

Now, let us use the following Lyapunov function:

$$
\bar{V}_{k}=z_{1_{k}}^{T}(P) z_{1_{k}}+S_{k}^{T} S_{k}
$$

where $0<P=P^{T} \in \mathfrak{R}^{(n-m) x(n-m)}$. So, the stability condition is

$$
\bar{V}_{k+1}-\bar{V}_{k}<0
$$

The sliding surface is

$$
S_{k}=G T^{-1} z_{k}=\bar{G} z_{k}=\left[\begin{array}{ll}
\bar{G}_{1} & \bar{G}_{2}
\end{array}\right]\left[\begin{array}{l}
z_{1_{k}} \\
z_{2_{k}}
\end{array}\right] \text {. }
$$

So,

$$
z_{2_{k}}=\left(\bar{G}_{2}\right)^{-1} S_{k}-\left(\bar{G}_{2}\right)^{-1} \bar{G}_{1} z_{1_{k}}
$$

Substituting (61) into (57) we have

$$
z_{1_{k+1}}=\overline{\bar{\Phi}}_{11} z_{1_{k}}+\overline{\bar{\Phi}}_{12} S_{k}+\bar{f}_{1_{k}}
$$

where $\bar{\Phi}_{11}=\bar{\Phi}_{11}-\bar{\Phi}_{12}\left(\bar{G}_{2}\right)^{-1} \bar{G}_{1}$ and $\bar{\Phi}_{12}=\bar{\Phi}_{12}\left(\bar{G}_{2}\right)^{-1}$.

Also,

$$
\begin{aligned}
S_{k+1}= & G x_{k+1}, \\
S_{k+1}= & G \Phi x_{k}+G \Gamma u_{k-H}+G f_{k}, \\
u_{k-H}= & F \underbrace{x_{k-H}}_{x_{k}-e_{k}}, \\
S_{k+1}= & G \Phi x_{k}-G \Gamma \underbrace{(G \Gamma)^{-1}[G(\Phi-I)+\bar{\beta} G]}_{-F} x_{k} \\
& +\underbrace{\left(f_{k}-\Gamma F e_{k}\right)}_{\widetilde{e}_{k}},
\end{aligned}
$$

$$
\begin{aligned}
& S_{k+1}=\underbrace{G x_{k}}_{S_{k}}-\bar{\beta} \underbrace{G x_{k}}_{S_{k}}+G \widetilde{e}_{k}, \\
& S_{k+1}=(1-\bar{\beta})\left(S_{k}+\overline{\bar{G}} \widetilde{e}_{k}\right),
\end{aligned}
$$

with $\overline{\bar{G}}=G /(1-\bar{\beta}), 0<\bar{\beta}<1.0$. 
Substituting (62) and (63) into (58)

$\bar{V}_{k+1}$

$$
\begin{aligned}
= & z_{1_{k+1}}{ }^{T}[P] z_{1_{k+1}}+S_{k+1}{ }^{T} S_{k+1} \\
= & \left(\overline{\bar{\Phi}}_{11} z_{1_{k}}+\overline{\bar{\Phi}}_{12} S_{k}+\bar{f}_{1_{k}}\right)^{T} \\
& \times(P)\left(\overline{\bar{\Phi}}_{11} z_{1_{k}}+\overline{\bar{\Phi}}_{12} S_{k}+\bar{f}_{1_{k}}\right) \\
& +(1-\bar{\beta})^{2}\left(S_{k}+\overline{\bar{G}} \widetilde{e}_{k}\right)^{T}\left(S_{k}+\overline{\bar{G}} \widetilde{e}_{k}\right) \\
= & z_{1_{k}}^{T} \overline{\bar{\Phi}}_{11}^{T} P \overline{\bar{\Phi}}_{11} z_{1_{k}}+z_{1_{k}}^{T} \overline{\bar{\Phi}}_{11}^{T} P \overline{\bar{\Phi}}_{12} S_{k} \\
& +z_{1_{k}}^{T} \overline{\bar{\Phi}}_{11}^{T} P \bar{f}_{1_{k}} \\
& +S_{k}^{T} \overline{\bar{\Phi}}_{12}^{T} P \overline{\bar{\Phi}}_{11} z_{1_{k}}+S_{k}^{T} \overline{\bar{\Phi}}_{12}{ }^{T} P \overline{\bar{\Phi}}_{12} S_{k} \\
& +S_{k}^{T} \bar{\Phi}_{12}^{T} P \bar{f}_{1_{k}} \\
& +\bar{f}_{1_{k}}^{T} P \overline{\bar{\Phi}}_{11} z_{1_{k}}+\bar{f}_{1_{k}}^{T} P \overline{\bar{\Phi}}_{12} S_{k}+\bar{f}_{1_{k}}^{T} P \overline{f_{1_{k}}} \\
& +(1-\bar{\beta})^{2}\left(S_{k}^{T} S_{k}+2 S_{k}^{T} \overline{\bar{G}}_{\widetilde{e}_{k}}+f_{k}^{T} \overline{\bar{G}}^{T} \overline{\bar{G}} \widetilde{e}_{k}\right),
\end{aligned}
$$

$\bar{V}_{k+1}$

$$
\begin{aligned}
& =z_{1_{k}}^{T} \overline{\bar{\Phi}}_{11}^{T} P \bar{\Phi}_{11} z_{1_{k}}+2 z_{1_{k}}^{T} \overline{\bar{\Phi}}_{11}^{T} P \overline{\bar{\Phi}}_{12} S_{k} \\
& +2 z_{1_{k}}^{T} \bar{\Phi}_{11}^{T} P \bar{f}_{1_{k}}+S_{k}^{T} \overline{\bar{\Phi}}_{12}^{T} P \overline{\bar{\Phi}}_{12} S_{k} \\
& +2 S_{k}{ }^{T} \overline{\bar{\Phi}}_{12}^{T} P \bar{f}_{1_{k}}+\bar{f}_{1_{k}}^{T} P \bar{f}_{1_{k}}+(1-\bar{\beta})^{2} S_{k}^{T} S_{k} \\
& +2(1-\bar{\beta})^{2} S_{k}^{T} \overline{\bar{G}} \widetilde{e}_{k}+(1-\bar{\beta})^{2} \widetilde{e}_{k}^{T} \overline{\bar{G}}{ }^{T} \overline{\bar{G}} \widetilde{e}_{k} \text {, } \\
& \bar{V}_{k+1}-\bar{V}_{k} \\
& =z_{1_{k}}^{T} \overline{\bar{\Phi}}_{11}^{T} P \overline{\bar{\Phi}}_{11} z_{1_{k}}-z_{1_{k}}^{T} P z_{1_{k}} \\
& +2 z_{1_{k}}^{T} \overline{\bar{\Phi}}_{11}^{T} P \overline{\bar{\Phi}}_{12} S_{k}+2 z_{1_{k}}^{T} \overline{\bar{\Phi}}_{11}^{T} P \bar{f}_{1_{k}} \\
& +S_{k}^{T} \overline{\bar{\Phi}}_{12}^{T} P \overline{\bar{\Phi}}_{12} S_{k}+2 S_{k}^{T} \bar{\Phi}_{12}^{T} P \bar{f}_{1_{k}} \\
& +\bar{f}_{1_{k}}^{T} P \bar{f}_{1_{k}}+(1-\bar{\beta})^{2} S_{k}^{T} S_{k}-S_{k}^{T} S_{k} \\
& +2(1-\bar{\beta})^{2} S_{k}^{T} \overline{\bar{G}} \widetilde{e}_{k}+(1-\bar{\beta})^{2} \widetilde{e}_{k}^{T} \overline{\bar{G}}^{T} \overline{\bar{G}} \widetilde{e}_{k}, \\
& \bar{V}_{k+1}-\bar{V}_{k} \\
& =z_{1_{k}}^{T} \underbrace{\left(\bar{\Phi}_{11}^{T} P \overline{\bar{\Phi}}_{11}-P\right)}_{-Q} z_{1_{k}}+2 z_{1_{k}}^{T} \overline{\bar{\Phi}}_{11}^{T} P \overline{\bar{\Phi}}_{12} S_{k}
\end{aligned}
$$

$$
\begin{aligned}
& +2 z_{1_{k}}^{T} \overline{\bar{\Phi}}_{11}^{T} P \bar{f}_{1_{k}}+S_{k}^{T} \overline{\bar{\Phi}}_{12}^{T} P \overline{\bar{\Phi}}_{12} S_{k} \\
& +2 S_{k}^{T} \overline{\bar{\Phi}}_{12}^{T} P \bar{f}_{1_{k}}+\bar{f}_{1_{k}}^{T} P \bar{f}_{1_{k}} \\
& +\left[(1-\bar{\beta})^{2}-1\right] S_{k}^{T} S_{k} \\
& +2(1-\bar{\beta})^{2} S_{k}^{T} \overline{\bar{G}} \widetilde{e}_{k}+(1-\bar{\beta})^{2} \widetilde{e}_{k}^{T} \overline{\bar{G}}^{T} \overline{\bar{G}} \widetilde{e}_{k}, \\
& \bar{V}_{k+1}-\bar{V}_{k} \\
& \leq-Q_{\min }\left\|z_{1_{k}}\right\|^{2}+2\left\|z_{1_{k}}\right\|\left\|\overline{\bar{\Phi}}_{11}^{T} P \overline{\bar{\Phi}}_{12}\right\|\left\|S_{k}\right\| \\
& +2\left\|z_{1_{k}}\right\|\left\|\overline{\bar{\Phi}}_{11}^{T} P\right\|\left\|\bar{f}_{1_{k}}\right\| \\
& +\left\|S_{k}\right\|\left\|\overline{\bar{\Phi}}_{12}{ }^{T} P \overline{\bar{\Phi}}_{12}\right\|\left\|S_{k}\right\| \\
& +2\left\|S_{k}\right\|\left\|\overline{\bar{\Phi}}_{12}{ }^{T} P\right\|\left\|\bar{f}_{1_{k}}\right\|+\underbrace{\|P\|}\left\|\bar{f}_{1_{k}}\right\|^{2} \\
& +\left|(1-\bar{\beta})^{2}-1\right|\left\|S_{k}\right\|^{2} \\
& +2(1-\bar{\beta})^{2}\left\|S_{k}\right\|\|\overline{\bar{G}}\|\left\|\tilde{e}_{k}\right\|+(1-\bar{\beta})^{2}\|\overline{\bar{G}}\|^{2}\left\|\tilde{e}_{k}\right\|^{2}, \\
& \bar{V}_{k+1}-\bar{V}_{k} \\
& \leq-Q_{\min }\left\|z_{1_{k}}\right\|^{2}+2 \delta\left\|z_{1_{k}}\right\|\left\|\overline{\bar{\Phi}}_{11}^{T} P \overline{\bar{\Phi}}_{12}\right\| \\
& +2 f_{1}\left\|z_{1_{k}}\right\|\left[\overline{\bar{\Phi}}_{11}^{T} P\right]+\delta^{2}\left\|\overline{\bar{\Phi}}_{12}{ }^{T} P \overline{\bar{\Phi}}_{12}\right\| \\
& +2 \delta f_{1}\left\|\overline{\bar{\Phi}}_{12}^{T} P\right\|+f_{1}^{2} P+\left|(1-\bar{\beta})^{2}-1\right| \delta^{2} \\
& +2 \delta\left(\bar{b}_{\max }\right)(1-\bar{\beta})^{2}\|\overline{\bar{G}}\|+(1-\bar{\beta})^{2}\left(\bar{b}_{\max }\right)^{2}\|\overline{\bar{G}}\|^{2},
\end{aligned}
$$

where $Q_{\min }$ denote the minimum singular value of $Q=$ $-\left(\overline{\bar{\Phi}}_{11}{ }^{T} P \overline{\bar{\Phi}}_{11}-P\right)$. Note that $\lim _{k \rightarrow \infty}\left\|\widetilde{e}_{k}\right\|=\bar{b}_{\max }<\infty$ and because the attractiveness condition (30) is satisfied we have $\left\|S_{k}\right\| \leq \delta<\infty$ for all $k$.

After some algebraic manipulation, (64) becomes

$$
\bar{V}_{k+1}-\bar{V}_{k} \leq-Q_{\min }\left(\left\|z_{1_{k}}\right\|-\frac{\delta_{1}}{Q_{\min }}\right)^{2}+\frac{\delta_{1}^{2}}{Q_{\min }}+\delta_{2},
$$

where

$$
\begin{aligned}
\delta_{1}= & \delta\left\|\overline{\bar{\Phi}}_{11}{ }^{T} P \overline{\bar{\Phi}}_{12}\right\|+f_{1}\left\|\overline{\bar{\Phi}}_{11}^{T} P\right\|, \\
\delta_{2}= & \delta^{2}\left\|\overline{\bar{\Phi}}_{12}^{T} P \overline{\bar{\Phi}}_{12}\right\|+2 \delta f_{1}\left\|\overline{\bar{\Phi}}_{12}^{T} P\right\|+f_{1}^{2}\|P\| \\
& +\left|(1-\bar{\beta})^{2}-1\right| \delta^{2}+2 \delta\left(\bar{b}_{\max }\right)(1-\bar{\beta})^{2}\left\|G_{\beta}\right\| \\
& +(1-\bar{\beta})^{2}\left(\bar{b}_{\max }\right)^{2}\left\|G_{\beta}\right\|^{2} .
\end{aligned}
$$


Therefore [19],

$$
\bar{V}_{k+1}-\bar{V}_{k}<0 \text { if }\left\|z_{1_{k}}\right\|>\frac{\delta_{1}+\sqrt{\delta_{1}{ }^{2}+Q_{\min } \delta_{2}}}{Q_{\min }}=r ;
$$

that is, the trajectory of $z_{1_{k}}$ will enter into ball with center at the origin and radius $r$ and will converge in finite time to the quasisliding mode band $\delta$. At steady state it stays in the neighborhood of the origin.

Proposition 5. Consider the uncertain system without delay of the form

$$
x_{k+1}=\Phi x_{k}+\Gamma v_{k}+f_{k},
$$

in which the sliding mode control law does not use predictors, that is,

$$
v_{k}=F x_{k}
$$

where $F=F_{e}+F_{n}, F_{e}=-(G \Gamma)^{-1} G(\Phi-I)$, and $F_{n}=$ $-(G \Gamma)^{-1} \bar{\beta} G, 0<\bar{\beta}<1.0$. So the attractiveness condition (30) is satisfied if $\left\|G x_{k}\right\|>\left\|G f_{k}\right\|$.

Proof. Taking into account the uncertainties $f_{k}$, (32) becomes

$$
\Delta S_{k+1}=G\left(\Phi x_{k}+\Gamma v_{k}+f_{k}\right)-G x_{k} .
$$

Substituting (69) into (70),

$$
\Delta S_{k+1}=-\beta G x_{k}+G f_{k} .
$$

In this case, there is no need for using predictor, and the proof is similar to the proof of Proposition 2, (40)-(42), with $\widetilde{e}_{k}=f_{k}$.

Note 5. Because $\left\|G f_{k}\right\|<\left\|G \widetilde{e}_{k}\right\|$ and Propositions 2,3, and 5, it can be concluded that the use of proposed control law (37) with state predictor (12) stabilizes the delayed system, however, increases the limits around the ideal equilibrium point. It means that uncertainties affect more the performance of the system when time delay is present.

In the following section, the presented propositions and notes are validated through a numerical example.

\section{Numerical Example: System of Second Order}

In this section, a system of order 2 to validate the propositions and notes presented in the previous section is utilized. Three cases are simulated and graphical results are presented through phase plans.

5.1. System Model and Design of Controllers. The uncertain model is used as follows:

$$
\begin{aligned}
\dot{x}_{1}(t)= & x_{2}(t), \\
\dot{x}_{2}(t)= & x_{1}(t)+\left[2+\Delta_{22} \operatorname{sen}(\pi t)\right] x_{2}(t) \\
& +u(t-\tau)+\Delta_{u} \operatorname{sen}(\pi t),
\end{aligned}
$$

where $x_{1}(t)$ and $x_{2}(t)$ are the states, $\Delta_{22}=4.0$ is the amplitude of the parametric uncertainty of the plant, and $\Delta_{u}=1.0$ is the amplitude of the disturbance in the control input. The time delay control input is $\tau=H \Delta, \Delta=2$ milliseconds is the sampling period, and $H$ is the actual number of delayed samples. It is considered that only a bounded range of $H$ is known. The estimated time delay $\widehat{\tau}$ of the predictors used in the control design is 40 milliseconds; that is, the number of estimated delayed samples $\widehat{H}$ is 20 . The initial states are $x_{1}(0)=0.2$ and $x_{2}(0)=0.2$. Note that this model without uncertainties has open-loop eigenvalues equal to " -0.4142 " and " +2.4142 "; thus, it represents an unstable plant.

The control law is generated through feedback of the states using the strategy DSMC, as follows.

(i) DSMC-P1 Controller:

$$
u_{k}=F \widehat{x}_{k} .
$$

The predicted state vector $\widehat{x}_{k}$ is obtained by means of (9). The matrix $F=F_{e}+F_{n}$ is the same as the one of DSMC-P2 described as follows:

(ii) DSMC-P2 Controller:

$$
u_{k}=F \widehat{x}_{k} .
$$

The predicted states vector $\widehat{x}_{k}$ is obtained by (12), $\widehat{x}_{k}=$ $\Psi^{(\widehat{H})} x_{k}$. The matrix $F=F_{e}+F_{n}$ is obtained according to (28), (36), and (37). The sliding surface is given by $S_{k}=G x_{k}$ and it is designed such that the sliding pole is the equivalent discrete of " -2 ", that is, " 0.9960 ". So

$$
\begin{aligned}
& G=\left[\begin{array}{ll}
996.0077 & 498.0050
\end{array}\right], \\
& F_{e}=\left[\begin{array}{ll}
-1.0000 & -3.9930
\end{array}\right], \\
& F_{n}=\left[\begin{array}{ll}
-49.8004 & -24.9002
\end{array}\right], \\
& \Psi^{(\widehat{H})}=(\Phi+\Gamma F)^{(20)}=\left[\begin{array}{cc}
0.9709 & 0.0239 \\
-1.2248 & 0.3107
\end{array}\right] .
\end{aligned}
$$

For $N=4,000$ sampling periods, that is, 8.0 seconds,

$$
\Psi^{(4,000)}=1.0 e-006\left[\begin{array}{cc}
0.1221 & 0.0048 \\
-0.2441 & -0.0095
\end{array}\right] ;
$$

for $N=10,000$ sampling periods, that is, 20.0 seconds,

$$
\begin{aligned}
\Psi^{(10,000)}= & 1.0 e-017\left[\begin{array}{cc}
0.4608 & 0.0180 \\
-0.9216 & -0.0359
\end{array}\right], \\
& \lim _{N \rightarrow \infty}\left\{\Psi^{(N)}\right\}=\left[\begin{array}{ll}
0 & 0 \\
0 & 0
\end{array}\right] .
\end{aligned}
$$

(iii) DSMC Controller:

$$
v_{k}=F x_{k},
$$

where $x_{k}$ is the actual state vector. The matrix $F=F_{e}+F_{n}$ is the same as the ones of DSMC-P2 and DSMC-P1. 


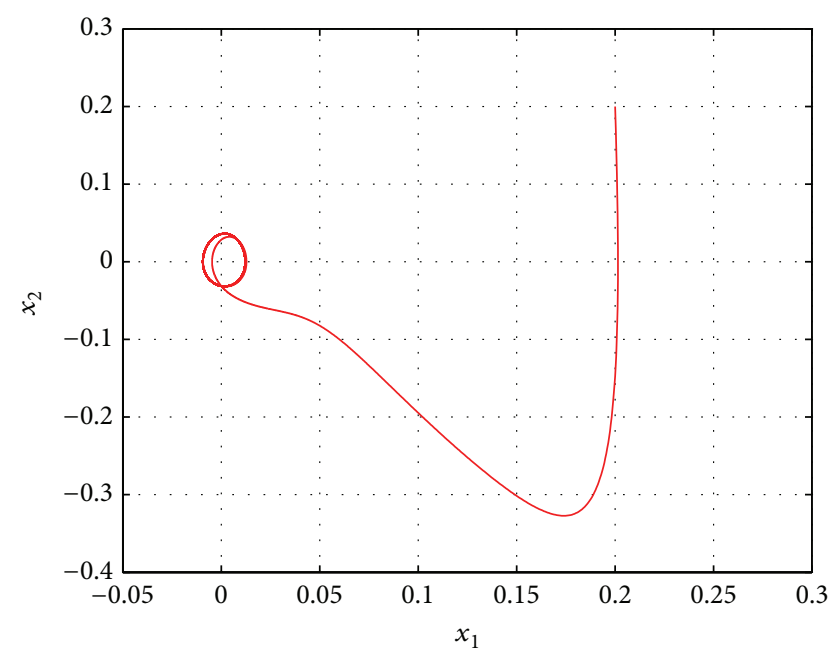

- DSMC

Figure 2: Phase plane for uncertain system without time delay, controlled by DSMC (without predictor).

5.2. Simulations and Analysis of Results. The computational simulations were performed using Matlab/Simulink software. Three cases are discussed as shown below.

Case (i). Uncertain system without time delay, controlled by DSMC.

In this case the uncertain system has no time delay, that is, $H=0$. The used controller is DSMC. The result is shown in Figure 2, where it can be noted that the controller has stabilized the system, taking the steady state trajectory in the neighborhood of the origin. Under the point of view of disturbances rejection, the system with DSMC controller had a good performance.

Case (ii). Uncertain system with time delay, controlled by DSMC (without predictor).

In this case the actual value $H$ is different at each simulation time interval as follows:

$$
\begin{aligned}
& T_{1}=\{0 \leq t \leq 20 \mathrm{~s}\}: \quad H=0 ; \\
& T_{2}=\{20 \mathrm{~s}<t \leq 40 \mathrm{~s}\}: \quad H=\widehat{H} ; \\
& T_{3}=\{40 \mathrm{~s}<t \leq 60 \mathrm{~s}\}: \quad H=1.5 \widehat{H} .
\end{aligned}
$$

DSMC without state predictor is used, that is, with the control law $v_{k}=F x_{k}$. Figure 3 shows the result. It may be noted that the system became unstable.

Case (iii). Uncertain system with time delay, controlled by DSMC-P1 or DSMC-P2.

Figure 4 shows the result for the system with the same time delay in the intervals $T_{1}, T_{2}$, and $T_{3}$, but now the plant is controlled by DSMC-P1 or DSMC-P2, that is, with predictor (9) or (12), respectively. Now the system is stable. This shows

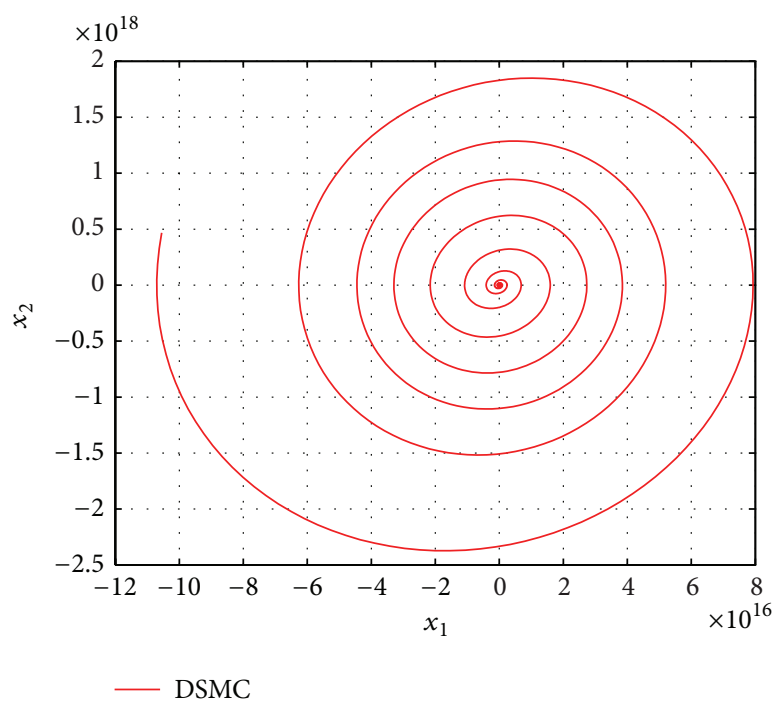

Figure 3: Phase plane for uncertain system with time delay, controlled by DSMC (without predictor).

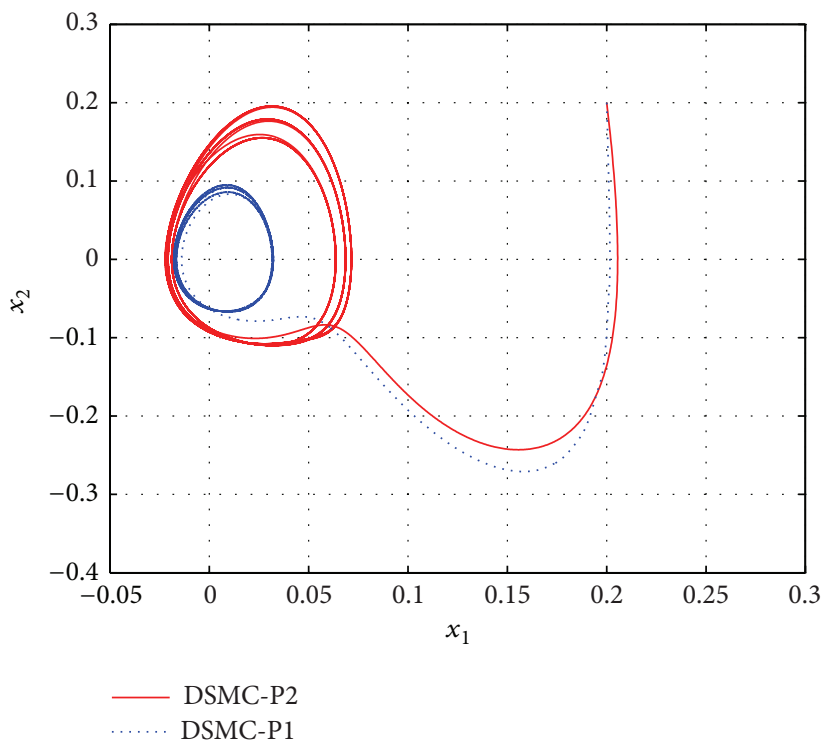

FIgURE 4: Phase plane for uncertain system with time delay, controlled by DSMC-P1 or DSMC-P2.

that this control strategy requires state predictor when time delay is present.

For each of the controllers, it can be seen that there are three different trajectories. From the smallest to the largest area, they represent the state trajectories for each time interval $T_{1}, T_{2}$, and $T_{3}$, respectively. The part " $\left(\sum_{i=0}^{H-1} \Psi^{-[i-(H-1)]}\right)(f)$ " of (53) increases as the delay is increased, implying a steady state error norm, $\lim _{k \rightarrow \infty}\left\|e_{k}\right\|=b_{\max }$, also higher at each interval $T_{1}, T_{2}$, and $T_{3}$. These results are in accordance with the Propositions 2, 3 , and 4 and also with Notes 3 and 4 . 
The best performance was obtained using the DSMCP1 controller, where the predictor is given by (9). However this predictor requires several past samples of the control signal, and, for each sampling period, many calculations are required. On the other hand, the DSMC-P2 controller also proved to be efficient. The main advantage is that it requires no previous samples of the control signal, and its computation, at each period, is very simple and quick.

It can be seen, by comparing Figures 2 and 4 that the delayed system controlled by DSMC-P1 or DSMC-P2 is stable, but it is more sensitive to disturbance. This fact is in accordance with Propositions 2 to 5 and Note 4.

\section{Conclusion}

This work approached uncertain systems with networked discrete-time sliding mode control (DSMC), subject to time delay. To minimize the degenerative effects of time delay, a simpler format of state predictor is used in the control law. The used state predictor has the advantage of fast computation, without the necessity of control signal sampling. The analyses and results from simulations showed the effectiveness of the proposed strategy with regard to the stabilization of uncertain time delay system, even in the presence of uncertainties and delays. The simulation results also confirm the analyses concerning the influence of the uncertainties in the networked control system performance with proposed DSMC strategy.

\section{Conflict of Interests}

The authors declare that there is no conflict of interests regarding the publication of this paper.

\section{Acknowledgments}

The authors thank CNPq and FAPESP, Process no. 2011/176100 , for the financial support.

\section{References}

[1] F. Lian, J. R. Moyne, and D. M. Tilbury, "Performance evaluation of control networks: ethernet, ControlNet, and DeviceNet," IEEE Control Systems Magazine, vol. 21, no. 1, pp. 66-83, 2001.

[2] B. Sharmila and N. Devarajan, "A survey-networked control systems," International Journal of Electrical Engineering, vol. 5, no. 6, pp. 757-768, 2012.

[3] J. P. Hespanha, P. Naghshtabrizi, and Y. Xu, "A survey of recent results in networked control systems," Proceedings of the IEEE, vol. 95, no. 1, pp. 138-172, 2007.

[4] H. C. Yi, H. W. Kim, and J. Y. Choi, "Design of networked control system with discrete-time state predictor over WSN," Journal of Advances in Computer Networks, vol. 2, no. 2, pp. 106109, 2014.

[5] M. Jungers, E. B. Castelan, V. M. Moraes, and U. F. Moreno, "A dynamic output feedback controller for NCS based on delay estimates," Automatica, vol. 49, no. 3, pp. 788-792, 2013.

[6] Y. I. Xu, K. Wang, Y. Shen, and J. Jian, "Robust $H_{\infty}$ networked control for uncertain fuzzy systems with time-delay," in
Advances in Electric and Electronics, vol. 155 of Lecture Notes in Electrical Engineering, pp. 115-124, Springer, Berlin, Germany, 2012.

[7] W. Ridwan and B. R. Trilaksono, "Networked control synthesis using time delay approach: State feedback case," International Journal on Electrical Engineering and Informatics, vol. 3, no. 4, pp. 441-452, 2011.

[8] M. Guinaldo, J. Sánchez, and S. Dormido, "Co-design strategy of networked control systems for treacherous network conditions," IET Control Theory and Applications, vol. 5, no. 16, pp. 1906-1915, 2011.

[9] W. Kim, K. Ji, and A. Ambike, "Networked real-time control strategies dealing with stochastic time delays and packet losses," in Proceedings of the American Control Conference (ACC '05), pp. 621-626, June 2005.

[10] Y. Tipsuwan and M. Chow, "Control methodologies in networked control systems," Control Engineering Practice, vol. 11, no. 10, pp. 1099-1111, 2003.

[11] F. Lian, J. Moyne, and D. Tilbury, "Network design consideration for distributed control systems," IEEE Transactions on Control Systems Technology, vol. 10, no. 2, pp. 297-307, 2002.

[12] R. A. deCarlo, S. H. Zak, and G. P. Matthews, "Variable Structure Control of Nonlinear Multivariable Systems: a Tutorial," Proceedings of the IEEE, vol. 76, no. 3, pp. 212-232, 1988.

[13] S. K. Spurgeon and R. Davies, "A nonlinear control strategy for robust sliding mode performance in the presence of unmatched uncertainty," International Journal of Control, vol. 57, no. 5, pp. 1107-1123, 1993.

[14] B. Draženović, "The invariance conditions in variable structure systems," Automatica, vol. 5, pp. 287-295, 1969.

[15] G. Xia and H. Wu, "Network-based neural adaptive sliding mode controller for the ship steering problem," in Advances in Swarm Intelligence, vol. 7928 of Lecture Notes in Computer Science, pp. 497-505, 2013.

[16] X. Han, E. Fridman, and S. K. Spurgeon, "Sliding mode control in the presence of input delay: a singular perturbation approach," Automatica, vol. 48, no. 8, pp. 1904-1912, 2012.

[17] Y. Yin, L. Xia, L. Song, and M. Qian, "Adaptive sliding mode control of networked control systems with variable time delay," in Electrical Power Systems and Computers, vol. 99 of Lecture Notes in Electrical Engineering, pp. 131-138, Springer, 2011.

[18] M. Yan, A. S. Mehr, and Y. Shi, "Discrete-time sliding-mode control of uncertain systems with time-varying delays via descriptor approach," Journal of Control Science and Engineering, vol. 2008, Article ID 489124, 8 pages, 2008.

[19] Y. Xia, G. P. Liu, P. Shi, J. Chen, D. Rees, and J. Liang, "Sliding mode control of uncertain linear discrete time systems with input delay," IET Control Theory and Applications, vol. 1, no. 4, pp. 1169-1175, 2007.

[20] K. Furuta, "Sliding mode control of a discrete system," Systems \& Control Letters, vol. 14, no. 2, pp. 145-152, 1990.

[21] S. Tarbouriech, I. Queinnec, T. Alamo, M. Fiacchini, and E. F. Camacho, "Ultimate bounded stability and stabilization of linear systems interconnected with generalized saturated functions," Automatica, vol. 47, no. 7, pp. 1473-1481, 2011. 


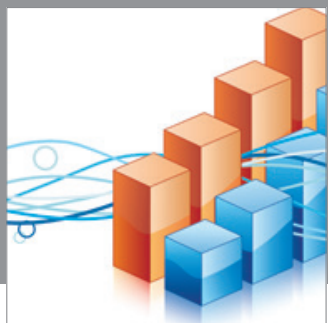

Advances in

Operations Research

mansans

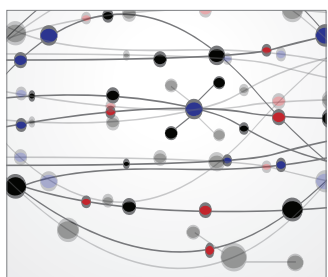

The Scientific World Journal
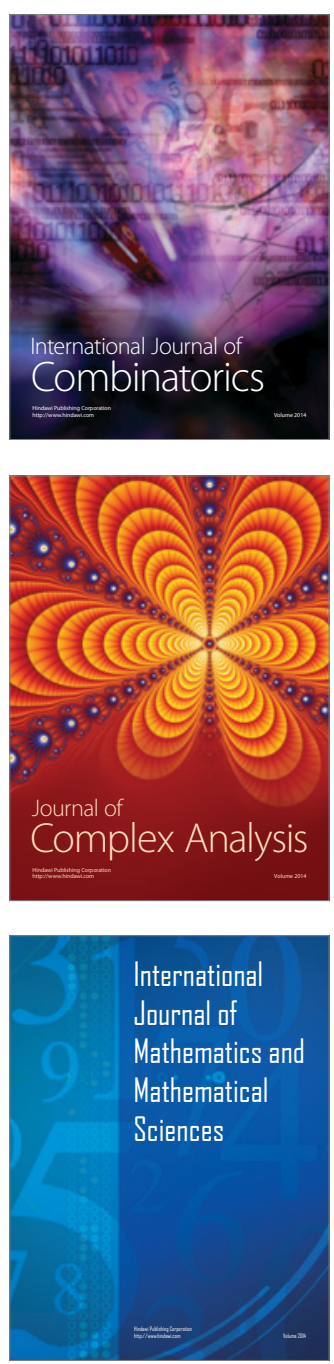
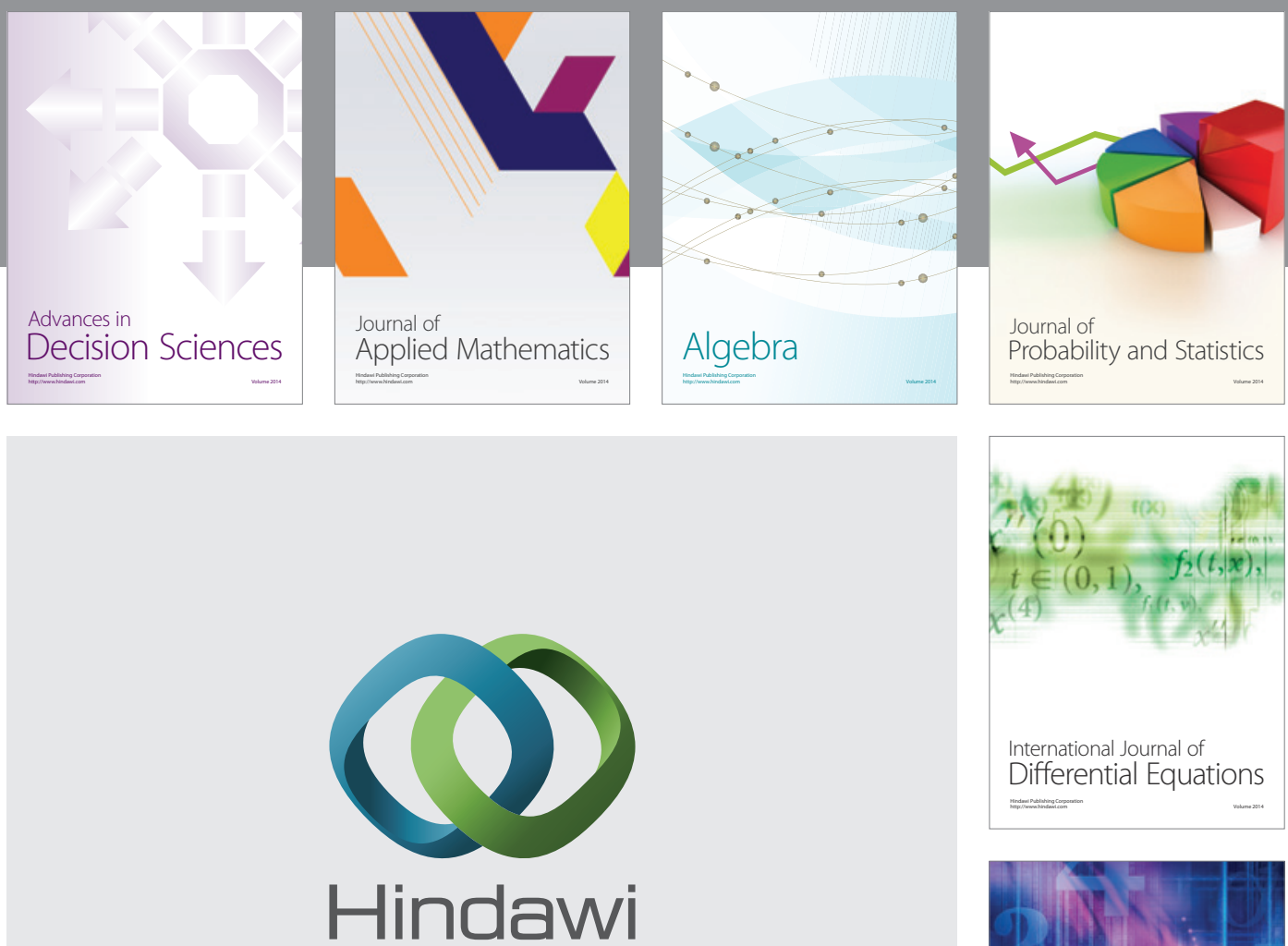

Submit your manuscripts at http://www.hindawi.com
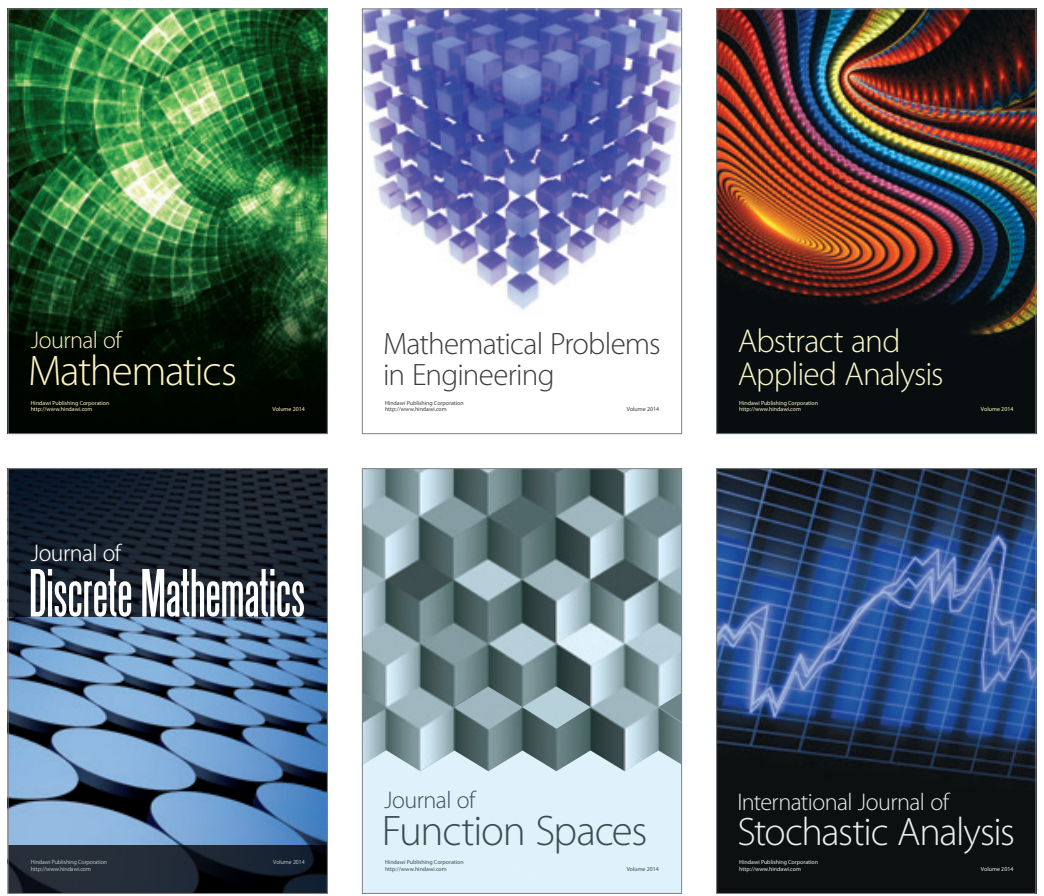

Journal of

Function Spaces

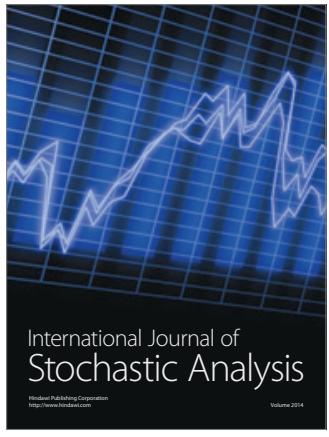

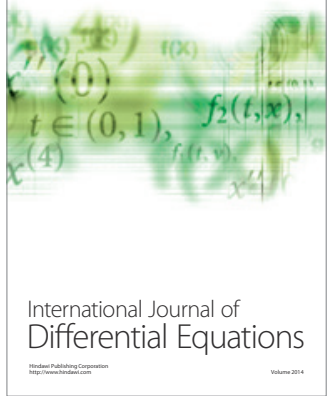
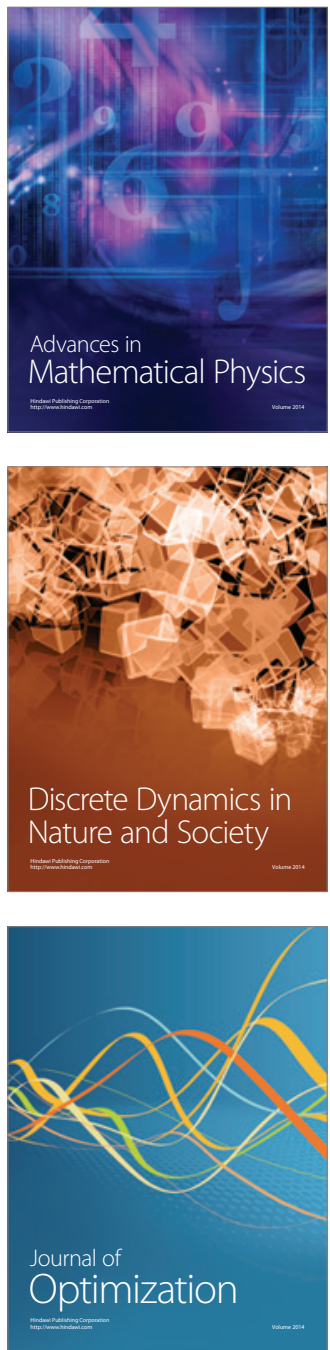